\title{
Analysis of Heart Rate and Respiratory Patterns in Sudden Infant Death Syndrome Victims and Control Infants
}

\author{
DAVID GORDON, DAVID P. SOUTHALL, DOROTHY H. KELLY, ADRIAN WILSON, \\ SOLANGE AKSELROD, JEAN RICHARDS, BARNEY KENET, ROBERT KENET, \\ RICHARD J. COHEN, AND DANIEL C. SHANNON
}

Pediatric Cardiology, University of Illinois at Chicago, University of Illinois Hospital, Chicago, Illinois [D.G.]; Pediatric Pulmonary Unit, Children's Service, Massachusetts General Hospital, Boston, Massachusetts [D.H.K., B.K., D.C.S.]; Department of Paediatrics, Cardiothoracic Institute, University of London, Brompton Hospital, England [D.P.S., J.R.]; Sheffield University and Area, Health Authority (Teaching), Royal Hallamshire Hospital, Sheffield, England [A.W.]; Department of Physics, Tel Aviv University, Ramat-Aviv, Israel [S.A.]; and HarvardMIT Division of Health Sciences and Technology and MIT Department of Physics, Massachusetts Institute of Technology, Cambridge, Massachusetts [R.J.C., R.K.]

\begin{abstract}
Retrospective analyses of patterns of breathing and heart rate variability obtained by visual inspection and spectral analysis of ECG and respiratory activity have provided markers associated with subsequent death in a referred population of infants at high risk for sudden infant death syndrome (SIDS). Such markers include breathing patterns characterized by excessive apneic pauses and periodic breathing, heart rate spectra characterized by increased low frequency oscillations, and respiratory activity spectra characterized by a widened "bandwidth" during regular breathing. To test whether such measurements could distinguish SIDS cases and randomly selected controls from a population study the data from 10 cases and 100 age-matched control subjects were analyzed blind. The code was disclosed after completion of the analysis. We found that none of the markers served to distinguish the SIDS cases from the controls in the population at large. This observation may indicate important physiological differences between infants destined to die in the referred high risk population and infants who die of SIDS at large. The possible reasons for our inability to identify the group of SIDS in the general population, as compared to the group of deaths in the referred high risk group are: (1) different disease processes in the two groups, (2) difference responses to the same disease process in the two groups, (3) a response reflecting the psychosocial setting of the referred high risk population, (4) methodological differences between this and previous studies. We conclude that these markers are not of value in screening the population at large. (Pediatr Res 20: 680-684, 1986)
\end{abstract}

Abbreviations

HR, heart rate

SIDS, sudden infant death syndrome

Received August 14, 195; accepted February 18, 1986.

Correspondence Dr. D. Gordon, Pediatric Cardiology, University of Illinois at Chicago, University of Illinois Hospital Box 6998, Chicago, IL 60680

Supported by the Reynolds Foundation, the Healthdyne Company, MESCO the McGraw Foundation, the National Foundation for Sudden Infant Death PhysioParameters, Inc., the Scholl Foundation, NSF Grant ECS8121571, Office of Naval Research Grant N00014-80-C-0520, USAF School of Aerospace Medicin Award F33615-84-C-0601, and NASA Grant NAG2-327. JMR was supported by the U.K. Foundation for the Study of Infant Death and DPS was supported by the British Heart Foundation.
Several patterns of breathing and heart rate variation have been identified in infants referred for evaluation for high risk of SIDS which appear to distinguish them from infants not at risk (1-3). High levels of periodic breathing and apneic pauses $(1,3$, 4) have been identified by visual inspection of respiratory activity recordings in the high risk infant referred for evaluation. Power spectrum analysis demonstrated two spectral variables that were increased in referred high risk babies who subsequently died suddenly, unexpectedly, and without explanation (2): the power of oscillation in heart rate with a period of approximately $15 \mathrm{~s}$ $(0.02-0.10 \mathrm{~Hz})$ and the bandwidth of the principal peak in the power spectrum of the respiratory activity during regular breathing.

It has previously been shown that there are two identifiable frequency bands in the heart rate spectra. The first of these is at low frequency $(0.02-0.1 \mathrm{~Hz})$ which contains information about both the $\beta$-adrenergic and parasympathetic moderators of the heart rate. The second is at the respiratory rate which contains information about the parasympathetic activity (5). Since only the low frequency power was found to be different between cases and controls from the previous study, these findings suggest that there is an increase in sympathetic activity in the cases.

Although both the visual inspection of breathing records and spectral analysis techniques appeared to identify infants at high risk, it was not clear whether they would be useful as a prospective population screening method nor was it clear whether previous results reflected the at risk state itself.

In the present study, we examined the hypothesis that (a) spectral measures of $\mathrm{HR}$ and respiration and (b) periodic breathing and apneic pauses would separate the SIDS infants from controls in the population at large.

\section{PATIENTS AND METHODS}

From a population-based study in which some results have already been reported (6), we examined the 24-h tape recordings of breathing movements and ECG of the first 10 full-term infants (12 recordings) (studied at around 6 wk of age) who died of SIDS and 100 full-term controls of similar age selected from the 6900 infants in the data base using a table of random numbers. Although the incidence of SIDS is about 1:500 we chose a 1:10 ratio of deaths to controls in order to increase the likelihood of identifying significant differences.

The mean age at recording of the infants who subsequently 
suffered SIDS was 6 wk (range 2-9 wk) and the mean time between the recording and death of the infants was $7 \mathrm{wk}$ (range $1-10 \mathrm{wk})$. The referred infants from the previous study (2) who subsequently suffered SIDS had a mean age of $7 \mathrm{wk}$ (range 2-18 wk) and the mean time between the recording and the death of the infant was $4 \mathrm{wk}$ (range 1-13 wk).

Recordings were made for $24 \mathrm{~h}$ using a Medilog 1 , four channel, battery operated tape recorder (Oxford Medical System) and were assigned code numbers so that the analysis could be performed blindly. A $60 \mathrm{~Hz}$ clock signal was also recorded to provide an accurate time base. The recording (6) and the analysis (5) techniques have been previously described.

We wished to compare the results of the analysis described herein with the results of the analysis obtained from infants at high risk of SIDS (2). For this comparison to be made, it was necessary to show that the data from the two studies were compatible. In both studies the ECG was collected in an identical manner. However, a major difference existed in obtaining the respiratory activity. In the previous study an impedance pneumography system was used, whereas the respiratory data in this present study were obtained from a pressure capsule transducer capsule positioned midway between the umbilicus and the xiphisternum. In order to investigate the possibility that the pressure transducer and impedance pneumography might yield respiratory signals with different spectral characteristics, respiratory activity was recorded in three babies using both transducers simultaneously. The mean frequency and bandwidth of respiratory activity were computed by the spectral technique described below in three regular breathing segments from each baby.

Oscillatory behavior was measured in two ways, by spectral analysis of $\mathrm{HR}$ and respiratory activity and by visual inspection of breathing records for periodic breathing and apneic pauses.

In order to select data for spectral analysis, each of the recordings on the infants included in the study was replayed and recorded onto chart paper for the period between midnight and $6 \mathrm{AM}$. The chart paper records were then examined visually and periods lasting longer than $5 \mathrm{~min}$ when the respiratory signal appeared regular in both amplitude and period were identified. If during that 5 -min period there were no rapid changes in the HR then those data were subjected to further analysis. In the event that no such episodes could be identified, the whole of the 24-h record was examined.

The ECG and respiratory signals were analyzed as follows (2, $5,7)$ : a computer algorithm identified the time of onset of each QRS complex in the ECG. A step-wise continuous instantaneous HR signal was computed whose magnitude was set to the reciprocal of the current interbeat interval for the duration of that interbeat interval. This signal was low pass filtered with a rectangular moving average window of $0.5 \mathrm{~s}$ width and then sampled at a frequency of $4 \mathrm{~Hz}$. The mean and linear trend of the resulting 1024 point time series was subtracted, the power spectrum computed from the absolute square of the fast Fourier transform of the time series, the spectrum smoothed with a five point Hanning window, and the spectrum corrected for the roll-off of the lowpass filter by dividing by

$$
\left(\frac{\sin 2 \pi \mathrm{fT}}{2 \pi \mathrm{fT}}\right)^{2}
$$

where $T=0.5 \mathrm{~s}$. The spectrum was divided by the square of the mean HR. The HR spectrum was only evaluated in the $0-1 \mathrm{~Hz}$ range. The respiratory activity signal was passed through a two pole $2 \mathrm{~Hz}$ antialiasing filter, sampled at $4 \mathrm{~Hz}$, and the power spectrum computed by squaring and smoothing the fast Fourier transform of the 1024 point time series as above.

Parameters derived from the HR and respiratory spectra included the mean and total variance for HR; the power of the HR oscillations at low frequency $(0.02-0.1 \mathrm{~Hz})$, the power of the oscillations at high frequency (respiratory frequency band), and the bandwidth of the principal peak in the respiratory power spectrum. In order to make the latter determination, each respiratory power spectrum was displayed at the same amplification and analyzed by eye for the width of the major peak above baseline noise (Fig. 1). For an individual recording, the power of the high frequency oscillations in HR was determined by identifying the band corresponding to the principal peak in the power spectrum of the respiratory signal and integrating over that same band the power spectral density of the HR.

In the above described manner, we analyzed between one and 18 data segments per infant. The value of the low frequency power in the HR power spectrum and the value of the respiratory bandwidth were identified for each segment so chosen. These two parameters distinguished SIDS from controls in a retrospective study (2). In order to avoid introducing bias, we selected from all the segments analyzed that segment with the lowest power in the low frequency band in the HR power spectrum.

Results from each baby were rank ordered giving equal weight to each of the two parameters from the selected data segment, by defining the ordering parameter, $\left(\mathrm{Z}_{\mathrm{i}}\right)$ as follows:

$$
Z_{\mathrm{i}}=1 / 2\left\{\frac{X_{\mathrm{i}}-\bar{X}}{\sigma_{x}}+\frac{Y_{\mathrm{i}}-\bar{Y}}{\sigma_{y}}\right\}
$$

Here $\bar{X}, \sigma_{x}$, and $X_{i}$ represent the mean, SD, and ith patient's value of low frequency $\mathrm{HR}$ power respectively. $\bar{Y}, \sigma_{y}$, and $Y_{i}$ represent the mean, $\mathrm{SD}$, and ith patient's value of respiratory bandwidth respectively. This method associates the highest rank order with the greatest power in oscillation of HR at low frequency and the greatest variability in respiration.

As an alternate statistical method, the data were entered stepwise into a computer-based cluster analysis program (Fastclus SAS) beginning with respiratory bandwidth and low frequency power in the HR spectrum and then including each of the other parameters. The data were forced into two or three clusters assuming there were two or three populations.

Increased variability of respiratory frequency and in particular, periodic breathing have also been associated with increased risk of SIDS $(1,3,4)$. All records from midnight to 6 AM were visually examined to identify respiratory patterns. Two trained observers analyzed each record visually and extracted the data according to a previously described method (1); interobserver variability was measured by one of us (DHK) and found to be $<5 \%$. The following measurements were made: regular breathing time, total minutes of periodic breathing, average duration of episodes of periodic breathing, and number of apneic pauses greater than $10 \mathrm{~s}$ in duration. Apneic pauses during periodic breathing in these infants rarely exceeded $8 \mathrm{~s}$. The Fastclus cluster

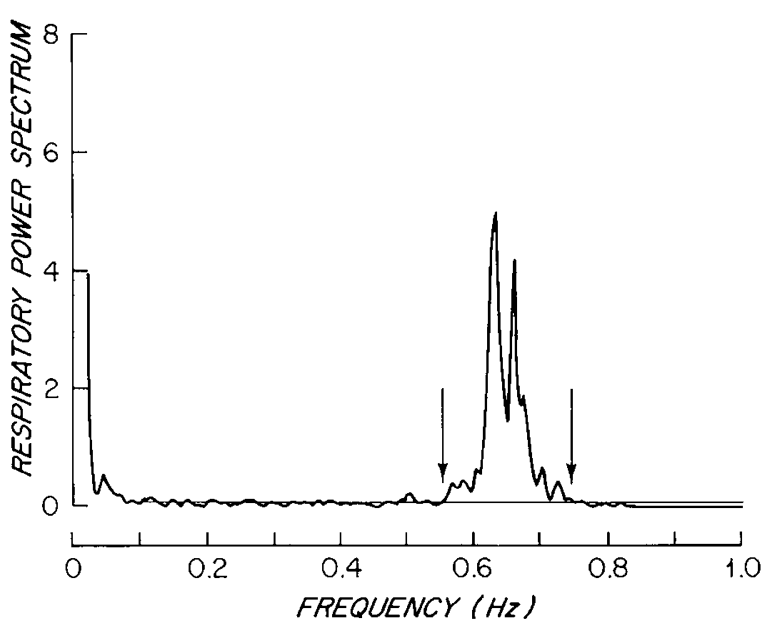

Fig. 1. Spectrum of respiratory activity. The respiratory band width is measured as a range of frequencies by dropping perpendiculars to the abscissa at the points where the spectrum rises above and falls below background noise. 
analysis program was used to identify clusters based on the above measured parameters. In addition, the data were rank ordered using two parameters, longest episode of periodic breathing, and apneic pauses of greater than $10 \mathrm{~s}$ duration, as described above for spectral analysis. For technical reasons one or more variables could not be statistically analyzed in five infants all of whom proved to be controls. Therefore, the analysis below refers only to those 95 controls in whom all variables could be analyzed.

The code was disclosed only after the above analyses were completed.

\section{RESULTS}

The comparison of the spectral composition between the impedance pneumography system and the pressure capsule transducer showed no significant differences and therefore comparisons between the results of this study and the previous one could be made.

By rank order, computed from respiratory bandwidth and low frequency HR oscillations, 14 infants appeared to be significantly different from our previous controls (1). However, these 14 infants included only one case of SIDS, a finding that was not greater than expected by chance alone. Analysis of two or three clusters using two, three, or four variables (low frequency power, respiratory bandwidth, the area of the respiratory peak in the HR power spectrum and mean HR) also failed to identify those infants who subsequently suffered SIDS.

The distribution of variables extracted from the time series of
HR and respiration were compared between control infants and the SIDS cases (Fig. 2). None of these distributions was significantly different either for all data or for data segregated by sex. (Kolmogorov-Smirnov, 2-tailed test for difference in distribution.)

We also compared the control and SIDS groups in the present general population study with those groups in the previous spectral analysis study (2) of a referred infant population. Using the two measures (respiratory bandwidth and low frequency HR oscillations), we found no statistical difference in the two control groups, but did find a significant difference between the two SIDS groups (Fig. 3).

Breathing patterns were evaluated over $6 \mathrm{~h}$ between midnight and 6 AM. On average $322 \mathrm{~min}$ were free of artifact and included in the analysis. The percent time with periodic breathing was 1.77 (SD 3.7) and the average number of apneic pauses $>10 \mathrm{~s} /$ 100 min was 1.02 (SD 2.75). Using multivariate analysis it was found that the percent time of periodic breathing, the average duration of episodes of periodic breathing, and the minutes of periodic breathing were highly and positively correlated with one another. Thus, in performing cluster analysis, only the clustering of the longest episode of periodic breathing and apneic pauses of $10 \mathrm{~s}$ or greater were examined (Fig. 4). Three clusters were identified with one containing a single subject as an outlier from the rest, a second containing 14 , and the remainder in a third cluster. This latter cluster contained all 36 infants who exhibited no periodic breathing. The subject with the extreme amount of
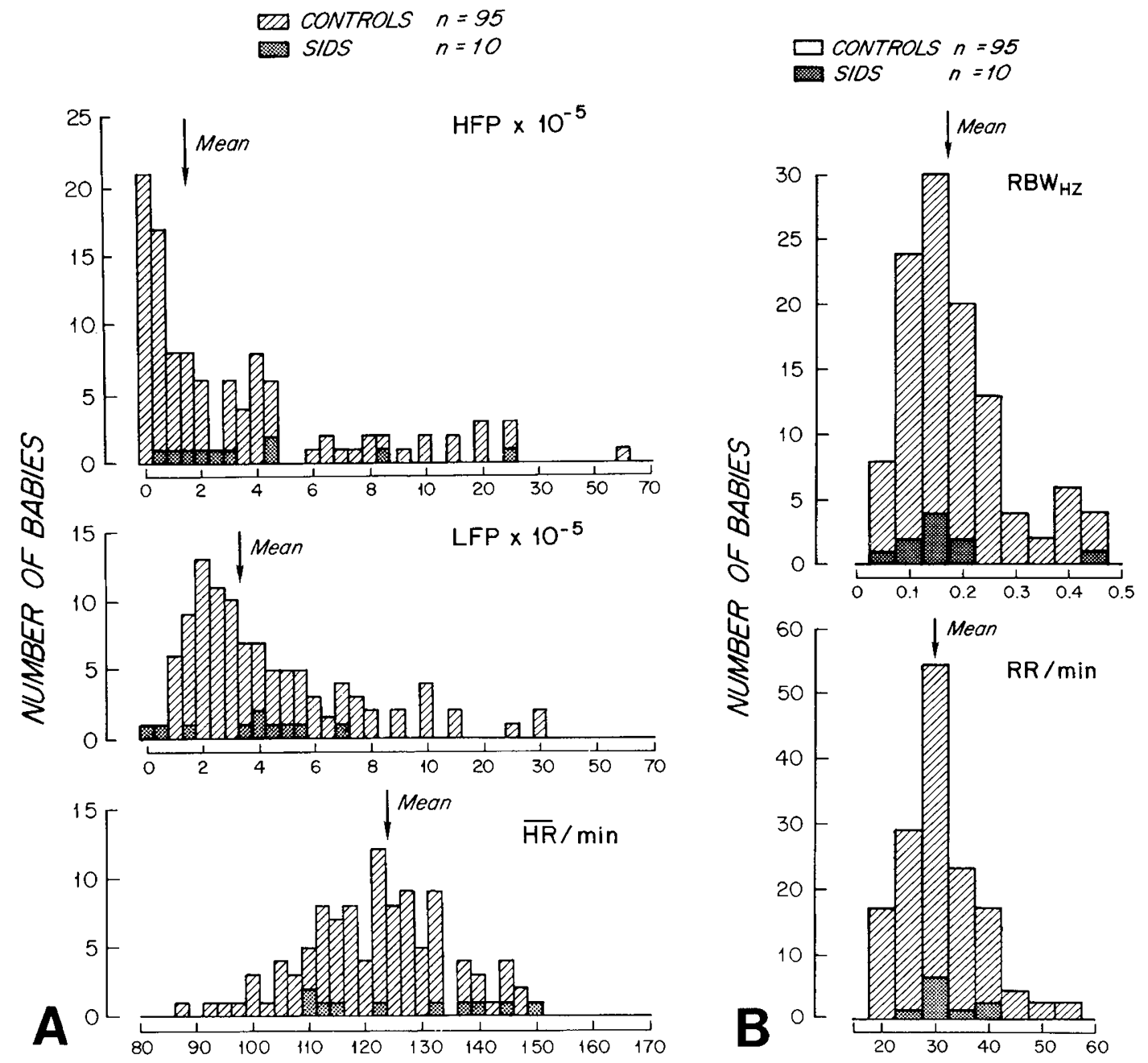

Fig. 2. The distributions of HR variables $(A)$ and respiratory variables $(B)$ derived from spectral analysis in Controls (hatched) and SIDS cases (stippled). $H F P$, high frequency power; $L F P$, low frequency power; $H R / \min$, mean $\mathrm{HR}$ (in beats/min); $R B W_{H z}$, respiratory band width (in $\mathrm{Hz}$ ); $R R / \mathrm{min}$, mean respiratory rate (in breaths $/ \mathrm{min}$ ). 


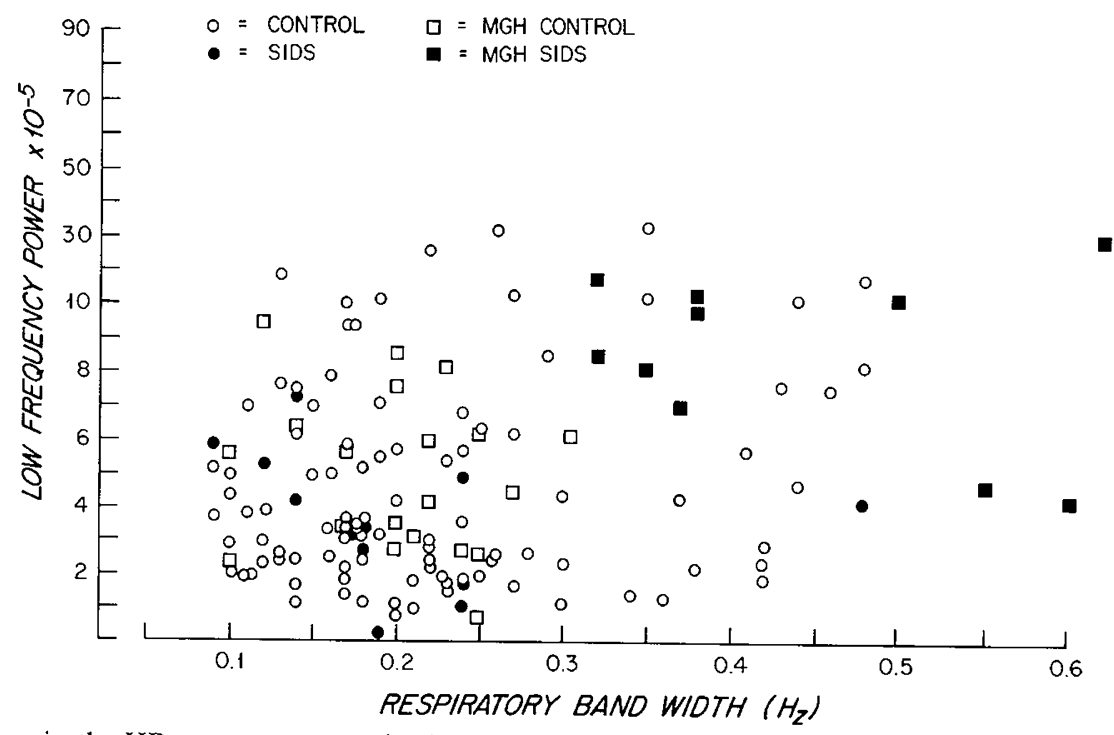

Fig. 3. Low frequency power in the HR power spectrum is plotted against the respiratory band width for infants in the previous study (square symbols) and the current study (circles). Deaths are indicated by closed squares and closed circles, respectively. Note that controls in the two studies have similar values while deaths in the previous study of high risk babies cluster in the Northeast quadrant.



Fig. 4. Cluster analysis of longest episode of periodic breathing and number of apneic spells greater than $10 \mathrm{~s}$ duration per $100 \mathrm{~min}$ of monitoring, in all subjects. There are three clusters: one of 14 subjects, a single outlier, and the remaining 90 subjects. The black dots represent the SIDS; the star represents the mean value of the cluster; the stippled area represents the extent of the cluster.

periodic breathing survived: one infant in the second cluster died of SIDS and nine of 85 in cluster three died of SIDS. Thus no significant differentiation of SIDS was obtained. Rank order computation also failed to identify SIDS cases.

After the code had been disclosed a further analysis of the data was performed. One major difference between this and the previous study (2) was that only healthy controls had been selected previously. Therefore, having broken the code, the results from the 100 controls were stratified according to each of the following variables: upper respiratory infection $(n=7)$, use of any medication $(n=14)$ (e.g. merbentyl, a parasympatholytic for colic), twin pregnancy $(n=3)$, Apgar score $<7$ at $1 \mathrm{~min}(n=$ 17), maternal smoking ( 12 greater than one pack per day and 24 less than one pack per day). Having excluded infants with at least one of these characteristics, 31 super-normals were identified and compared to the SIDS infants using the spectral-derived variables. Possible systematic effects of these factors on spectral variables were also examined. This analysis did not improve the separation of SIDS from controls nor were the spectral variables in any of the subsets different from the super-normals.

While sampling data, it was observed that for 12 of the infants it had been necessary to examine the entire $24-\mathrm{h}$ recording in order to find regular breathing segments. To determine whether this variation in selecting data segments might have biased the results, they were placed in rank order. Seven babies fell above the median rank and five fell below. Moreover, these babies were not disproportionately represented among SIDS versus controls or among any subset of controls.

\section{DISCUSSION}

Previous studies carried out in referred infants at high risk for SIDS have identified patterns of HR variation and respiration which appear to be markers of risk (8). A previous study (2) identified two spectral measures-enhanced oscillations in the $\mathrm{HR}$ at frequencies between 0.02 and $0.10 \mathrm{~Hz}$ and increased respiratory signal bandwidth-which appeared to distinguish high risk infants who subsequently died from controls. Other studies $(1,3,4)$ have presented evidence that increased variability of respiration evidenced by apneic pauses and periodic breathing are characteristics of the at-risk state.

In both this and the previous study (2) we have selected data for analysis by selecting periods when the respiratory waveform was regular in both amplitude and rate. Clearly, it would have been better to select data on the basis of "sleep state." However, it is impossible to directly classify sleep state from recordings containing only the ECG and respiratory waveform since this requires the EEG, EMG, and EOG. It is highly likely that our measures will be affected by sleep state. However, by selecting the data segment with the lowest power in the low frequency band we should have reduced the effects of choosing data segments from different sleep states on the results reported.

The purpose of the present study was to establish whether these markers that appear to be predictors of risk in a preselected referred population might also be predictors of risk in the population at large.

We find that these markers are not of predictive value in the population at large and thus should not be of utility in population screening for SIDS.

The lack of correlation of the respiratory and HR variation measures with the sudden deaths in the general population, as compared with the sudden deaths in referred infants thought to be at high risk, suggests important physiological differences be- 
tween the two SIDS populations. These differences may reflect: (1) different disease processes in the two groups, (2) different responses to the same disease processes in the two groups, or (3) a response reflecting the psychosocial setting of the referred high risk group.

It may be that the disease process, and in fact the pathophysiological mechanism of death itself, differs between the two groups. For example, we could speculate that the referred infants suffer from a primary respiratory control defect clinically manifested by "near-miss" episodes and choking spells. In fact in the previous spectral study (2) in five of the eight cases there was at least one near miss episode and in two others choking spells were present. The markers of breathing and HR variation that correlate with risk may possibly also reflect this primary respiratory control defect.

Another interpretation of our results is that the referred population constitutes a preselected subset of SIDS who suffer one or many near-miss events (which may or may not be observed) as opposed to the SIDS in the at large population who might suffer only one fatal episode. The respiratory and HR variation markers may reflect the sequelae of these many near-miss episodes in the referred population rather than reflecting the underlying pathophysiological defect itself.

Finally, the referred population is exposed to severe psychosocial stresses. It may be that the markers that correlate with death reflect an interplay between this stress and underlying pathophysiology, which does not occur in the completely unexpected SIDS in the population at large.

In conclusion, the abnormal pattern of $H R$ variation and respiration established as markers of SIDS in the referred highrisk infant population do not appear to be predictive of SIDS in the population at large and therefore do not appear suitable for population screening. However, these markers may still be useful in the evaluation of the referred high-risk population itself. It remains to be determined (9) whether new methods of analysis of ECG and respiratory records may be of predictive value for SIDS in the population at large.

Acknowledgments. The authors acknowledge the technical support of Ronald Berger, David Carley, Bryan Nesmyth, and Oswald Williams, the secretarial support of Mrs. Diana Minasian and the support of members of the Pediatric Pulmonary Laboratory, Massachusetts General Hospital. We especially thank the British Heart Foundation, the U.K. Foundation for the Study of Infant Death, and the people of Brighton and Doncaster who supported the collection of the data used in this study.

\section{REFERENCES}

1. Kelly DH, Shannon DC. 1979 Periodic breathing in infants with near-mis sudden infant death syndrome. Pediatrics 62:355-360

2. Gordon D, Cohen RJ, Kelly DH, Akselrod S, Shannon DS. 1984 Sudden infant death syndrome: abnormalities in short term fluctuations in heart rate and respiratory activity. Pediatr Res 18:921-926

3. Steinschneider A, Weinstein S, Diamont E. 1982 The sudden infant death syndrome and apnea/obstruction during neonatal sleep and feeding. Pediatrics $70: 858-863$

4. Moore SE, Walsh JK, Kennen WJ, Farrell MK, Wolske SR, Kramer M. 1981 Periodic breathing in infants with histories of prolonged apnea. Am J Dis Child 135:1029-1031

5. Akselrod S, Gordon D, Ubel FA, Shannon DC, Barger AC, Cohen RJ. 198 Power spectrum analysis of heart rate fluctuations: a quantitative probe of beat-to-beat cardiovascular control. Science 213:220-222

6. Multi-Centered Study Group: Southall DP, Richards JM, de Swiet M, Arrowsmith WA, Cree JE, Fleming PJ, Orme RL'E, Radford MJ, Wilson AJ, Shannon DC, Alexander JR, Brown NJ, Shinebourne EA 1983 Identification of infants destined to die unexpectedly during infancy: evaluation of predictive importance of prolonged apnea and disorders of cardiac rhythm or conduction. Br Med J 286:1091-1096

7. Pomeranz B, Macaulay RJB, Caudill MA, Kutz I, Adam D, Gordon D, Barger AC, Shannon DS, Cohen RJ, Benson H. 1985 Assessment of autonomic function in man by heart rate spectral analysis. Am J Phys 248:H151-H153

8. Oren $\mathbf{J}$, Kelly DH, Shannon DC. Identification of high risk group for SIDS among infants who were resuscitated for sleep apnea, Pediatrics (in press)

9. Kitney RI. 1984 New findings in the analysis of heart rate variability in infants. Automedica 5:289-310 